\title{
Numerical Study on Statistical Behaviors of Two-Dimensional Dry Foam
}

\author{
Yunchang Seol ${ }^{1}$ and Yongsam Kim²,* \\ ${ }^{1}$ Department of Applied Mathematics, National Chiao Tung University, \\ 1001 Ta Hsueh Road, Hsinchu 300, Taiwan. \\ 2 Department of Mathematics, Chung-Ang University, Dongjakgu Heukseokdong, \\ Seoul 156-756, Korea.
}

Received 4 December 2017; Accepted (in revised version) 15 February 2018

\begin{abstract}
We investigate the statistical behaviors of two-dimensional dry foam using numerical simulations based on the immersed boundary (IB) method. We model the liquid phase of a foam as a thin elastic boundary with surface tension and the gas phase as a viscous incompressible fluid which can go through the liquid boundary. We present evidence of the existence of a limiting scaling state of the dry foam dynamics in which the asymptotic value of $\mu_{2}$, the second moment of the distribution of the numbers of cell sides, lies in the range of $1.3 \pm 0.3$. We also numerically verify some well-known formulas derived in the dynamics of two-dimensional dry foam such as von Neumann relation, Lewis law, and Aboav-Weaire law. Our simulation results are comparable to those of soap froth experiments and Potts model simulations. Furthermore, we investigate the statistical behaviors of two-dimensional dry foam in an oscillatory shear flow to show the applicability of our method to more general flow conditions.
\end{abstract}

PACS: 02.50.Ng, 05.50.+q, 47.55.nb, 83.80.Iz

Key words: Foam dynamics, immersed boundary method, scaling state, von Neumann relation, Lewis law, Aboav-Weaire law.

\section{Introduction}

Foam or foam-like material is ubiquitous in daily life and in industry, appearing in such forms as a pint of beer, soda water, shaving cream, dish washer solution, and fire extinguisher. Liquid foam is a gas-filled space divided into cells with liquid boundaries. The thin liquid boundaries move under the influence of surface tension and allow the surrounding gas to flow through the boundaries. Thus the capillarity of the cell boundaries

${ }^{*}$ Corresponding author. Email addresses: ycseol@math.nctu.edu.tw (Y. Seol), kimy@cau.ac.kr (Y. Kim) 
and gas exchange between cells together result in the evolution of the size of the cells and the topological structure of a foam [1]. This process is called diffusive coarsening.

The diffusive coarsening of a two-dimensional dry foam obeys a simple dynamical law called von Neumann relation $[2,3]$ for the evolution of cells. Let $A_{n}$ be the area of a cell with $n$ sides, its rate of change can be determined as follows

$$
\frac{d A_{n}}{d t}=k(n-6)
$$

where $k$ is the diffusion constant proportional to both the permeability and the surface tension. The von Neumann relation simply says that the area is constant for six-sided cells, a cell with fewer than six sides tends to shrink (and in fact reaches zero area in finite time), and a cell with more than six sides tends to grow. If the von Neumann relation alone governed the coarsening process of a foam, the problems related to its dynamics would be trivial; however, when a cell shrinks and finally disappears, its neighboring cells might increase or decrease their number of sides, resulting in topological changes in the foam structure, and thus the rates of area change of these cells increase or decrease.

The statistics of two-dimensional foams has been widely investigated using numerical simulations and experimental studies, see [1,4-7] and the references therein. A typical coarsening process begins with a nearly regular array of hexagonal cells and gradually evolves into a completely disordered pattern. The principal quantity used to characterize such disordered cellular structure is $\mu_{2}$, as defined later, the second moment of the distribution $\rho(n)$ of the number of cells with $n$ sides. It is known that there exists a scaling state in which the distribution $\rho(n)$ is almost time-invariant with a fixed range of the second moment $\mu_{2}=1.5 \pm 0.3$. The topological disorder of foam structure including the time-invariant $\rho(n)$ has been extensively studied experimentally [8,9] and computationally [10-12], just to name a few.

There have been many numerical modelings for the evolution and scaling state of a foam. In $[1,6,13,14]$, the authors simulated the evolution of a two-dimensional dry foam within the framework of the following assumptions: Laplace-Young condition, Plateau's rule, and the von Neumann relation (1.1). Potts model uses a quasi-microscopic metallurgical view of foam structure in which the interior of each cell is composed of a collection of lattices [1]. Potts model has been widely used to simulate the cellular structure as an extension of the Ising model, and its applicability to the coarsening process of soap froth in a steady state has been well demonstrated in $[15,16]$. There are other foam simulations that take the fluid dynamics into account based on a boundary integral formulation $[17,18]$. More numerical works on two- and three-dimensional dry or wet foams can also be found in $[19,20]$ and the references therein.

In [21], the authors introduced an immersed boundary (IB) method [22] to simulate the fluid dynamics of a two-dimensional dry foam by modeling the gas phase of the foam as a viscous incompressible fluid and the liquid phase as a massless network of permeable internal boundaries with surface tension. The gas diffusion through the liquid phase of the foam was modeled by allowing the internal boundaries to slip relative to the 
fluid, at a velocity (speed and direction) proportional to the boundary force [21,23].

When a foam evolves in time, it goes through topological changes in its structure. The 'T1' process occurs when an edge of a cell gradually shrinks and is about to form a quadruple junction. Since a quadruple junction is unstable, after some transient, this vertex decomposes to form two triple junctions, but in a different configuration. The 'T2' process occurs when a three-edged cell gradually shrinks and finally disappears. Other topological changes are also possible through the combination of these two processes. In [24], the authors introduced the T1 and T2 processes to the IB computation for the foam dynamics in order to produce topological changes of a cell network.

In this paper, we use the same computational approaches as introduced in $[21,24]$ to investigate the statistical behaviors of two-dimensional dry foams. After we briefly describe the IB method and its numerical scheme for the two-dimensional foam dynamics including the topological changes in Section 2, we present the numerical results on the statistical behaviors of two-dimensional foams in Section 3. First, we show that there exists a limiting scaling state in which the distribution $\rho(n)$ of the number of cells with $n$ sides is almost time-invariant with a fixed range of the second moment $\mu_{2}$. In addition, we verify some well-known statistical findings on a two-dimensional dry foam; namely, (a) the exponent of growth for average cell area over time, (b) von Neumann law which describes the rate of change of the area of $n$-sided cells with respect to time, (c) Lewis law which expresses the relation between the average area of $n$-sided cells and the number of cell side $n$, and (d) Aboav-Weaire law which describes the change of the average number of sides of neighbors of $n$-sided cells in terms of the number of cell sides. We compare our numerical results with those of soap froth experiments and Potts model simulations. Finally, we explore the statistical behaviors of two-dimensional dry foams under an oscillatory shear flow, thus requiring the full fluid dynamics treatment of the present paper. Summary and conclusions are presented in Section 4.

The purpose of the present paper is two-fold. The first one is to show that our numerical method based on the IB method can be a proper tool to deal with the foam dynamics, which is accomplished by investigating the statistical behaviors of the foam structure in a static flow. The second purpose is to show that the present numerical method can be applied to the foam dynamics in more general flow conditions. Since we treat the full fluid dynamics as well as the motion of foam structure, we can easily extend our model to the case in which non-equilibrium flows significantly occur within each cell of the foam. For this purpose, we explore the statistical behaviors of two-dimensional dry foams under an oscillatory shear flow.

\section{Model equations and implementation}

Consider a two-dimensional viscous incompressible fluid (gas) in a domain $\Omega$ that is partitioned into cells by the internal boundary of foam. The internal foam boundary, denoted by $\mathbf{X}(s, t)$, interacts with the surrounding gas which can diffuse through the internal foam 
boundary. Then the equations of motion can be summarized as follows:

$$
\begin{aligned}
& \rho\left(\frac{\partial \mathbf{u}}{\partial t}+\mathbf{u} \cdot \nabla \mathbf{u}\right)=-\nabla p+\mu \nabla^{2} \mathbf{u}+\mathbf{f}, \\
& \nabla \cdot \mathbf{u}=0, \\
& \mathbf{F}(s, t)=\frac{\partial}{\partial s}(\gamma \boldsymbol{\tau}), \\
& \mathbf{f}(\mathbf{x}, t)=\int \mathbf{F}(s, t) \delta(\mathbf{x}-\mathbf{X}(s, t)) d s \\
& \frac{\partial \mathbf{X}}{\partial t}(s, t)=\mathbf{u}(\mathbf{X}(s, t), t)+M \mathbf{F} /\left|\frac{\partial \mathbf{X}}{\partial s}\right| \\
& \quad=\int \mathbf{u}(\mathbf{x}, t) \delta(\mathbf{x}-\mathbf{X}(s, t)) d \mathbf{x}+M \mathbf{F} /\left|\frac{\partial \mathbf{X}}{\partial s}\right| .
\end{aligned}
$$

Eqs. (2.1) and (2.2) are the fluid equations for a viscous incompressible fluid, in which the unknowns are the fluid velocity $\mathbf{u}(\mathbf{x}, t)$, the fluid pressure $p(\mathbf{x}, t)$, and the force per unit area applied by the internal foam boundary to the fluid $\mathbf{f}(\mathbf{x}, t)$, where $\mathbf{x}=(x, y)$ are fixed Cartesian coordinates, and $t$ is the time. The constant parameters $\rho$ and $\mu$ are the fluid density and viscosity, respectively.

Eq. (2.3) determines the force density of the internal foam boundary where $\tau(s, t)=$ $\frac{\partial \mathbf{X}}{\partial s} /\left|\frac{\partial \mathbf{X}}{\partial s}\right|$ is the unit tangent vector of the foam boundary and the constant $\gamma$ is the surface tension. Eq. (2.4) changes Lagrangian force density $\mathbf{F}(s, t)$ into Eulerian one $\mathbf{f}(s, t)$, and Eq. (2.5) is the equation of motion of the internal foam boundary where $M$ is the permeability constant. The first term on the right-hand side of Eq. (2.5) expresses the no-slip condition that the internal foam boundary moves at the local fluid velocity, and the second term describes the normal slip motion of the foam boundary relative to the fluid. This normal slip is a consequence of the permeability of the foam boundary, see [21] for a detailed derivation of the normal slip due to the boundary permeability. Note that Eqs. (2.4) and (2.5) both involve the two-dimensional Dirac delta function $\delta(\mathbf{x})=\delta(x) \delta(y)$, which expresses the local character of the interaction between the fluid and the immersed internal foam boundary.

For the numerical implementation to solve the system (2.1)-(2.5), we use a standard first-order IB method, generalized to take a permeable foam boundary into account [21, 23]. The step-by-step procedure of the numerical implementation used here can be summarized as follows (see [21] for a greater detail):

(a) Compute the Lagrangian force density using the position of the internal foam boundary, which is done by the discretization of Eq. (2.3).

(b) Distribute this force density defined at Lagrangian grid into the force density at Eulerian spatial grid to be applied in the Navier-Stokes equations. This is done by a discretization of Eq. (2.4).

(c) Solve the discretized version of the fluid equations (2.1)-(2.2) with the Eulerian force density to update the velocity field and pressure. 
(a)

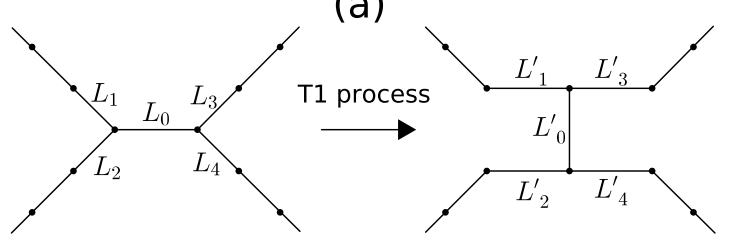

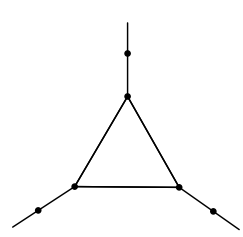

(b)

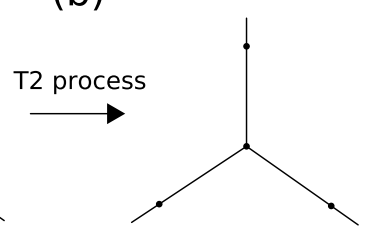

Figure 1: T1 process (a): when the length of a cell edge is smaller than the critical length $L_{\mathcal{c}}$, we rotate the edge by 90 degrees about its center, break the old connections, and make new ones as shown in (a). T2 process (b): when the lengths of the edges of a 3-edged cell are all smaller than the critical length $L_{c}$, we replace the 3-edged cell by one vertex at the centroid of the cell, and connect the new vertex directly to the three edges which previously formed triple junctions with the 3-edged cell.

(d) Update the foam boundary position which moves at the local fluid velocity of the updated velocity field with correction for relative slip. This is done by approximating Eq. (2.5).

At the end of each time step, we redistribute the internal foam boundary points to maintain reasonable resolution along the boundary. A proper resolution of the internal foam boundary is maintained by the following two processes: (1) if the distance between two neighboring boundary points is larger than $h / 2$ where $h$ is the spatial meshwidth of fluid, we add a new boundary point halfway between them; and (2) if the distance between two neighboring boundary points is smaller than $h / 4$, we delete both points and create a new boundary point halfway between them.

In addition to the redistribution of the internal boundary points, we perform topological changes when they are needed at the end of each time step. Since quadruple or higher-order junctions are unstable, they should be resolved into triple junctions, which induces topological changes. The topological changes of a 2D foam network are composed of two primary topological processes [1,6,24]: (1) removal of an edge between two neighboring cells, so that they cease to be neighbors, and its replacement by an edge between two other cells that consequently become neighbors ('T1' process); and (2) the removal of a three-sided cell ('T2' process), see Fig. 1.

The T1 process is needed when an edge of a cell gradually shrinks and is about to form a quadruple junction. When the length $L_{0}$ of an edge is smaller than a critical length $L_{c}$, we rotate the edge by 90 degrees about its center, break the old connections, and make new ones by connecting the rotated edge to the other four edges which previously formed the two triple junctions, as shown in Fig. 1(a). However, we make the actual switch only when it lowers the total potential energy, i.e., $\sum_{i=1}^{4} L_{i} \geq \sum_{i=1}^{4} L_{i}^{\prime}$. Otherwise, we do not make the switch (T1 process fails).

The T2 process is the disappearance of a 3-edged cell when three vertices of the cell become one point (vertex). When the lengths of three edges of a three-edged cell are all smaller than the critical length $L_{c}$, we replace the three-edged cell by one vertex at the centroid of the cell, and connect the new vertex directly to the three edges which previously formed triple junctions with the three-edged cell, see Fig. 1(b). 


\section{Statistics of foam dynamics}

In this section, we introduce the physical and computational parameters used in our numerical experiments and present the simulation results on the statistical behaviors of two-dimensional dry foams. By comparing our computational results with those of soap froth experiments and Potts model simulations, both of which were well reported in [5, $15,25]$, we shall show that the present numerical method is a proper tool to investigate the foam dynamics.

In our numerical simulations, we choose the computational domain $\Omega=[0,1] \times[0,1]$ $\mathrm{cm}^{2}$ with periodic boundary conditions, and construct a Voronoi network in the domain as the initial configuration of a foam composed of 1200 cells. We first define the distribution (frequency) of the number of cells with $n$ edges, $\rho(n)$, as the number of the $n$-edged cells over the total number of cells which compose the foam structure. Then the second moment $\mu_{2}$ of the side distribution can be defined as

$$
\mu_{2}=\sum \rho(n)(n-\langle n\rangle)^{2},
$$

where $\langle n\rangle$ is the average number of sides of the cells, which can be shown to be 6 by Euler's theorem [1]. The second moment $\mu_{2}$ of the side distribution indicates the complexity or disorder of the foam structure. In order to investigate the effect of the initial complexity or disorder of a foam structure on the foam dynamics, we initially construct the foam with various values of $\mu_{2}$, see the left panels of Fig. 2 .

Throughout this paper, we use the mesh width $h=\Delta x=\Delta y=1 / 1024 \mathrm{~cm}$, which is uniform and fixed in time, and the time step duration $\Delta t=1.5625 \times 10^{-4} \mathrm{~s}$. The fluid density is $\rho=10^{-3} \mathrm{~g} / \mathrm{cm}^{3}$, and the viscosity is $\mu=2 \times 10^{-3} \mathrm{~g} /(\mathrm{cm} \mathrm{s})$. The surface tension $\gamma$ used in Eq. (2.3) is fixed at $2.4 \times 10^{-2} \mathrm{erg} / \mathrm{cm}$, and the permeability $M$ in Eq. (3.3) is $3.0 \times 10^{-4}$ $\mathrm{cm}^{2} /$ (dyne s). These two values determine the diffusion constant $k=\frac{\pi}{3} \gamma M \approx 7.54 \times 10^{-6}$ $\mathrm{cm}^{2} / \mathrm{s}$ in Eq. (1.1) which is almost the same as the value used in previous experiments [26].

Fig. 2 shows the motion of the foams with three different values of the initial second moment: $\mu_{2}=0.6$ (top), 1.2 (middle), and 1.8 (bottom). The selected times are $t=0,320 \mathrm{~s}$, and $640 \mathrm{~s}$ from left to right. As time goes on, the cells with fewer than six edges shrink and disappear, and the cells with more than six edges grow in area according to the von Neumann law (1.1). Thus, the overall result of these changes in area is the diffusive coarsening of the foam structure. In the course of the coarsening, the foams experience topological changes via T1 and T2 processes, reducing the total number of cells. Compare the configurations of the foams at the initial and later times. Note that, even though the initial complexity of the three foams are different, i.e., the foam in the panel (g) is more disordered than the other two foams in the panels (a) and (d), the three foams seem to be similarly disordered at later times, see the foam structures in the middle and right columns. 
(a)

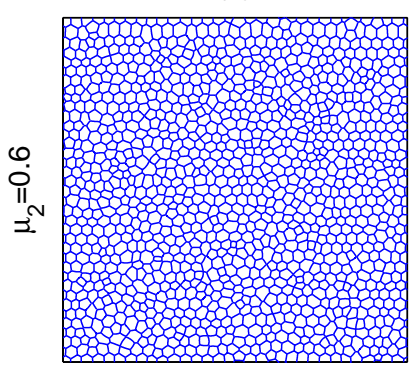

(d)

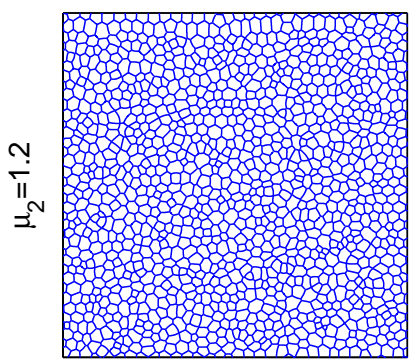

(g)

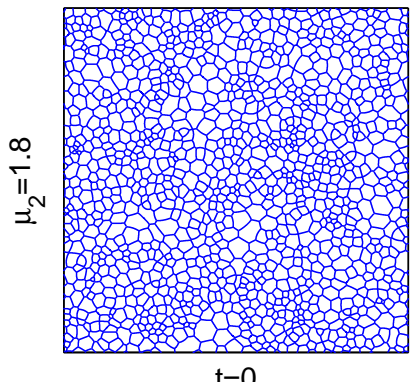

(b)

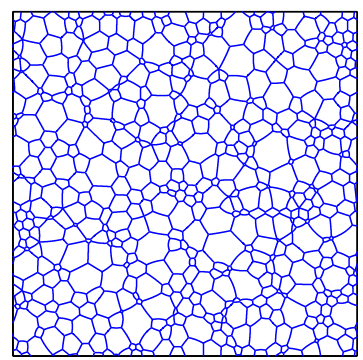

(e)

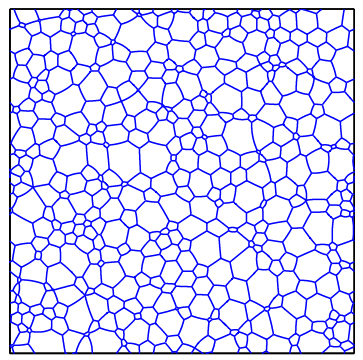

(h)

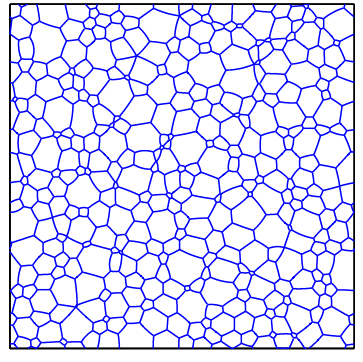

$t=320 \mathrm{~s}$ (c)

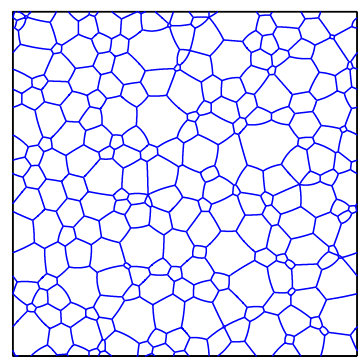

(f)

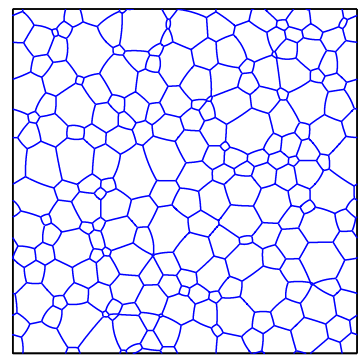

(i)

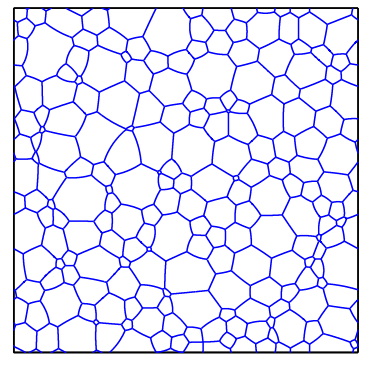

$\mathrm{t}=640 \mathrm{~s}$

Figure 2: The motion of the foams with three different values of the initial second moment $\mu_{2}$ at some selected times: $t=0,320 \mathrm{~s}$, and $640 \mathrm{~s}$ from left to right. The initial second moments are $\mu_{2}=0.6(\mathrm{a}, \mathrm{b}, \mathrm{c}), 1.2(\mathrm{~d}, \mathrm{e}, \mathrm{f})$, and $1.8(\mathrm{~g}, \mathrm{~h}, \mathrm{i})$. We can observe the coarsening behavior of the foam networks. Even though the second moment $\mu_{2}$, which represents the complexity of a foam structure, is initially different in the three cases, three foams seem to be similarly disordered at later times.

\subsection{Average area and side distribution}

A foam goes through diffusive coarsening of its structure and topological changes to reduce the total number of cells over time as shown in Fig. 2. To be more quantitative, we show in Fig. 3(a) the total number of cells as functions of time for the three different initial second moments: $\mu_{2}=0.6$ (line with $\triangle$ ), 1.2 (line with $\square$ ), and 1.8 (line with $\bigcirc$ ). As expected, the total numbers of cells decrease in all three cases, which indicates the coarsening process of the foams. As the initial $\mu_{2}$ gets larger, the decrement of the total number of cells is faster initially for some time. Later after about $t=500 \mathrm{~s}$, however, the 
(a)

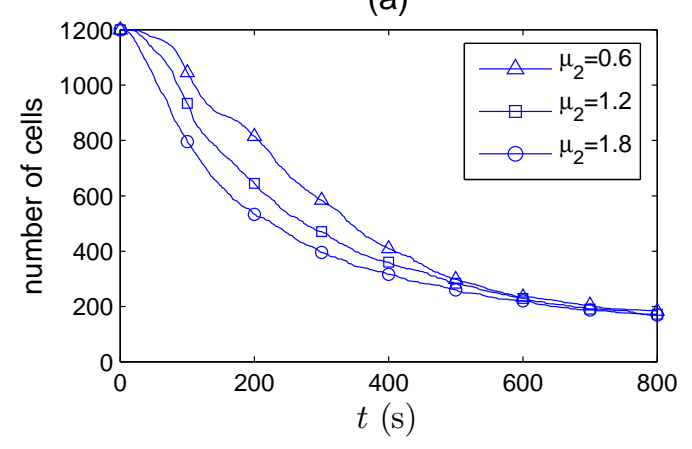

(b)

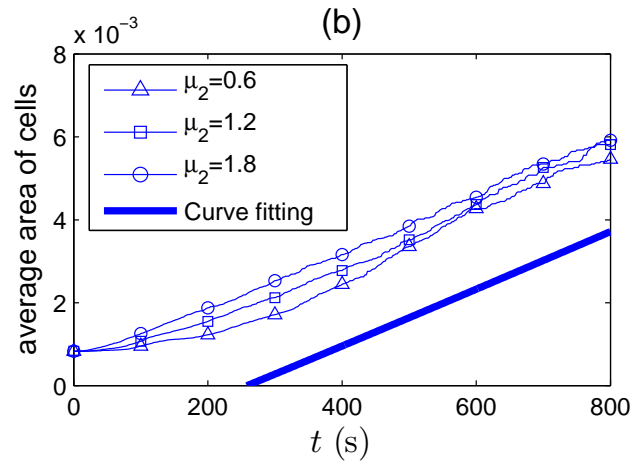

Figure 3: The total number (a) and the average area (b) of cells as functions of time. The total number of cells decreases as time goes on, with the decrement being faster initially as the initial second moment gets larger. Independent of the initial second moment, the average area of cells approaches a linear growth in time after when the foams are considered to be fully disordered.

decrement gets slower for all three cases, and the total numbers of the cells in the three foams become almost the same.

We investigate the change of average area of the cells in a foam as time evolves. Since the total area of a foam in our model is equal to that of the computational domain which is $1 \mathrm{~cm}^{2}$, the average area of cells $\langle A\rangle$ can be computed as 1 divided by the total number of cells. Fig. 3(b) shows the average area of cells as functions of time for three different cases: $\mu_{2}=0.6$ (line with $\triangle$ ), 1.2 (line with $\square$ ), and 1.8 (line with $\bigcirc$ ). We also compute the least square approximation $\langle A\rangle_{l}$ of the area function $\langle A\rangle$ which is fitted to a function of the following form:

$$
\langle A\rangle_{l}=C_{1} t^{\alpha}+C_{2}
$$

where $C_{1}$ and $\alpha$ are constants to be determined, and $C_{2}$ is the initial value of the average area.

When the coarsening dynamics of a foam is governed by the von Neumann's relation (1.1), the exponent is known to be $\alpha=1$ at the scaling state in which the distribution function $\rho(n)$ is almost constant $[2,3,27,28]$. We can see from Fig. 3(b) that, after some transient, the average areas $\langle A\rangle$ of the three foams seem to be all linear in time. Compare the three graphs of the area function $\langle A\rangle$ and the bold line which represents a linear function with the exponent $\alpha=1$. In fact, the exponents of the least square approximation $\langle A\rangle_{l}$ of the area function $\langle A\rangle$ are $\alpha=1.0369$ for the initial $\mu_{2}=1.8$ after $t=100 \mathrm{~s}, \alpha=1.0464$ for the initial $\mu_{2}=1.2$ after $t=200 \mathrm{~s}$, and $\alpha=1.0625$ for the initial $\mu_{2}=0.6$ after $t=400 \mathrm{~s}$. At each of these times, each of the three foams might be considered to reach a fully disordered foam and a limiting scaling state which shall be discussed now.

Since von Neumann relation (1.1) describes the rate of change of area for each individual cell in dry two-dimensional foam, it also bears on how the average area $\langle A\rangle$ of cells changes with time. It was shown theoretically and experimentally in [29] that, once a limiting scaling state is reached, the ratio $\langle A\rangle^{2} /\left\langle A^{2}\right\rangle$ is constant, where $\left\langle A^{2}\right\rangle$ is the av- 
(a)

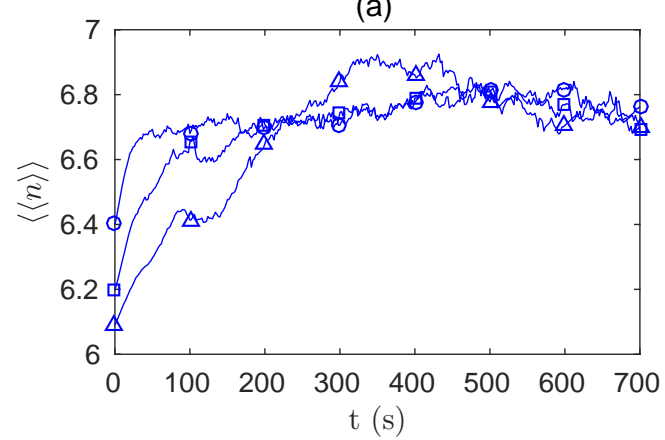

(c)

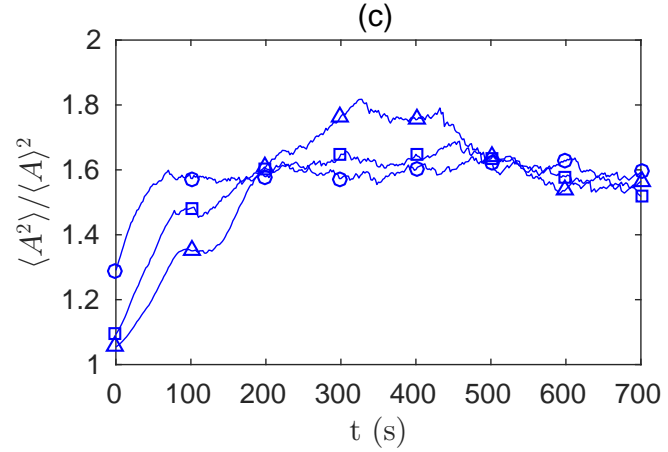

(b)
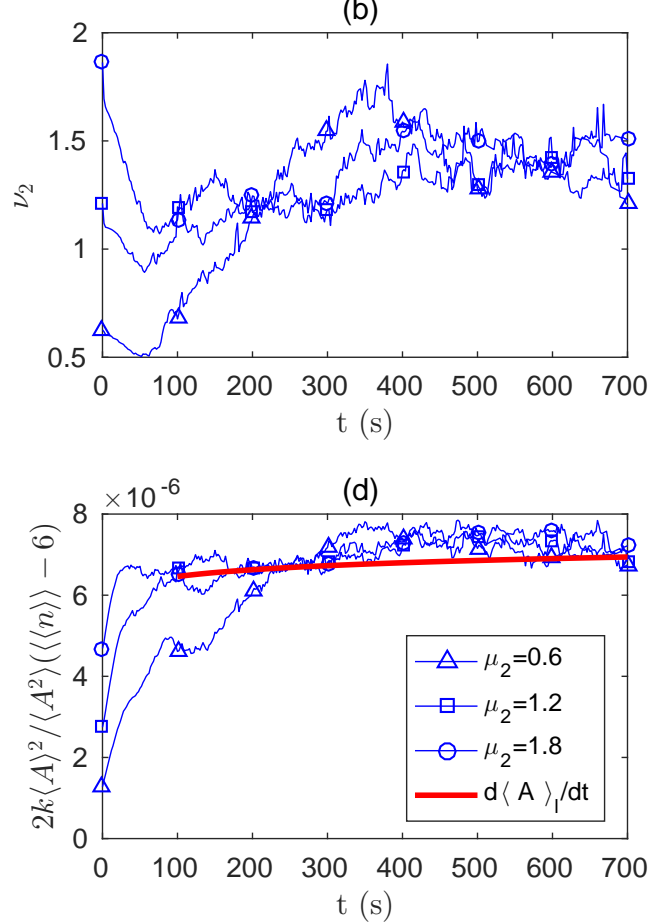

Figure 4: The area-weighted average number of sides, $\langle\langle n\rangle\rangle=\sum n F(n)(\mathrm{a})$, its second moment, $v_{2}=\sum(n-$ $\langle\langle n\rangle\rangle)^{2} F(n)(\mathrm{b})$, the ratio $\langle A\rangle^{2} /\left\langle A^{2}\right\rangle$ (c), and the comparison between $\frac{d\langle A\rangle_{l}}{d t}$ and the right-hand side of Eq. (3.3) as functions of time for the three different initial second moments: $\mu_{2}=0.6$ (line with $\triangle$ ), 1.2 (line with $\square$ ), and 1.8 (line with $\bigcirc$ ).

erage square area of cells, and thus the rate of change of the average area is governed by the following formula:

$$
\begin{aligned}
\frac{d\langle A\rangle}{d t} & =2 k \frac{\langle A\rangle^{2}}{\left\langle A^{2}\right\rangle} \sum F(n)(n-6) \\
& =2 k \frac{\langle A\rangle^{2}}{\left\langle A^{2}\right\rangle}(\langle\langle n\rangle\rangle-6),
\end{aligned}
$$

where $F(n)$ is the total area of the $n$-sided cells which represents the probability that a randomly chosen point in space lies inside an $n$-sided cell. The quantity $\langle\langle n\rangle\rangle=\sum n F(n)$ is the area-weighted average number of sides which is different from the average number of sides of the cells $\langle n\rangle=\sum n \rho(n)=6$ and, the difference of $\langle\langle n\rangle\rangle$ from 6 plays an important role in the evolution of the foam.

Fig. 4(a) and (b) show the area-weighted average number of sides, $\langle\langle n\rangle\rangle$, and its second moment, $v_{2}=\sum(n-\langle\langle n\rangle\rangle)^{2} F(n)$, as functions of time, respectively, for the three different initial second moments: $\mu_{2}=0.6$ (line with $\triangle$ ), 1.2 (line with $\square$ ), and 1.8 (line with $\bigcirc$ ). Since the cells with a larger number of sides tend to have greater area, the area-weighted 
average number of side $\langle\langle n\rangle\rangle$ is larger than 6 . After $t=400 \mathrm{~s},\langle\langle n\rangle\rangle=6.802 \pm 0.123$, and its second moment $v_{2}=1.438 \pm 0.231$, which are comparable to the range of values observed in [29].

We can see from Fig. 4(c) that the ratio $\langle A\rangle^{2} /\left\langle A^{2}\right\rangle$ approaches almost the same constant 1.6 after $t=400 \mathrm{~s}$ for the three initial second moments. This is argued in [29] and used in the derivation of Eq. (3.3). Fig. 4(d) compares the left-hand and right-hand sides of Eq. (3.3) in which the rate of change of the average area $\frac{d\langle A\rangle}{d t}$ is computed using Eq. (3.2), i.e., $\frac{d\langle A\rangle_{l}}{d t}=C_{1} \alpha t^{\alpha-1}$. One can see that, once the limiting scaling state is reached after about $t=400 \mathrm{~s}$, the difference between $\frac{d\langle A\rangle_{l}}{d t}$ (solid line) and the left-hand side of Eq. (3.3) is small for the three initial second moments, which confirms the formula (3.3).

Since the side distribution $\rho(n)$ and the second moment $\mu_{2}$ represent the complexity or disorder of a foam structure, they are important statistical data and thus have been investigated by many researchers [4,30-33]. It has long been claimed that, as a foam evolves, it reaches a limiting scaling state in which the two statistical quantities $\rho(n)$ and $\mu_{2}$ are almost time-invariant. After Smith [4] observed a limiting scaling state with $\mu_{2}=1.296$ in his experimental work with a two-dimensional disordered soap froth in 1952, many experimental and numerical studies have been done to verify the existence of a limiting scaling state in which the steady second moment $\mu_{2}$ is around either 1.2 or 1.4-1.5 [30-33].

Fig. 5(a) shows the second moment $\mu_{2}$ as functions of time for three different initial second moments: $\mu_{2}=0.6$ (line with $\triangle$ ), 1.2 (line with $\square$ ), and 1.8 (line with $\bigcirc$ ). We can see that, after some transient, the second moment $\mu_{2}$ oscillates around some fixed constant value which is about 1.3 for all three cases. We performed more computational experiments with different initial second moments (data not shown here) and found similar behaviors. That is, the second moment $\mu_{2}$ stays around $\mu_{2}=1.3 \pm 0.3$ which is consistent with the range of values observed in [30-33]. This observation that the second moment $\mu_{2}$ stays in some fixed range confirms the existence of a limiting scaling state.

When the second moment $\mu_{2}$ oscillates in some fixed range, we also find that the side distribution $\rho(n)$ is almost time-invariant. The lower panels of Fig. 5 depict the side distribution $\rho(n)$ at $t=0(\mathrm{~b})$ and $t=520 \mathrm{~s}$ (c) for the three different initial second moments: $\mu_{2}=0.6$ (line with $\triangle$ ), 1.2 (line with $\square$ ), and 1.8 (line with $\bigcirc$ ). Even though the side distributions $\rho(n)$ are initially different at $t=0(\mathrm{~b})$, they behave similarly at $t=$ $520 \mathrm{~s}$ (c) and at other times in the limiting scaling states. Our simulation results are also comparable to those obtained in soap froth experiments (is) and in the Potts model simulations $(\times)$ [15]. Especially, the peak of the side distribution $\rho(n)$ occurs when the number of sides is equal to $n=5$ [15] or 6 [33]. Note that the side distribution $\rho(n)$ shown in Fig. 5 is almost time-invariant, as we plotted it at more different times (not shown here). This observation together with the second moment $\mu_{2}$ staying in some fixed range confirms that the foam approaches a limiting scaling state with asymptotic values of side distribution $\rho(n)$ and second moment $\mu_{2}$, regardless of initial complexity of the foam structure. 
(a)

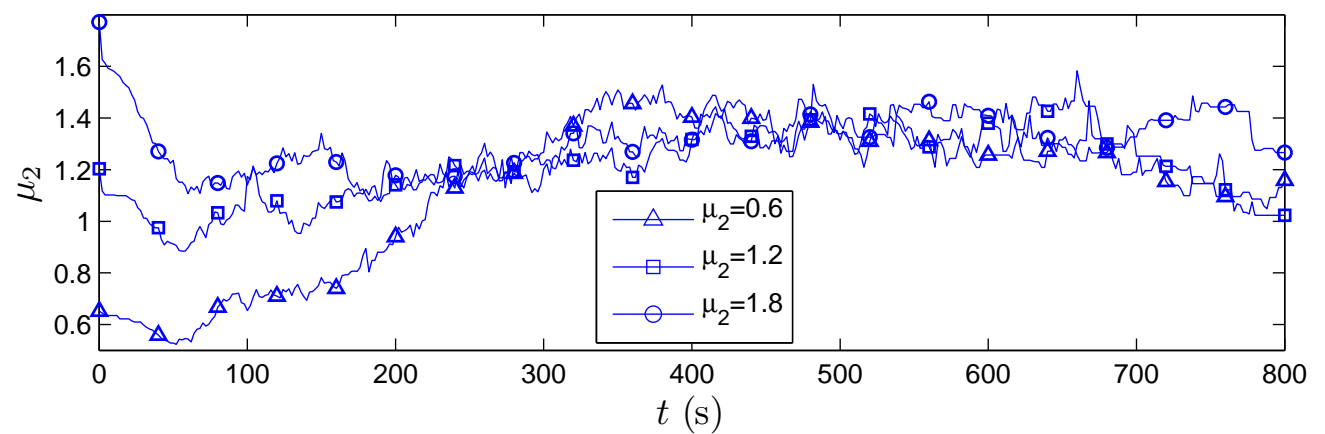

(b)

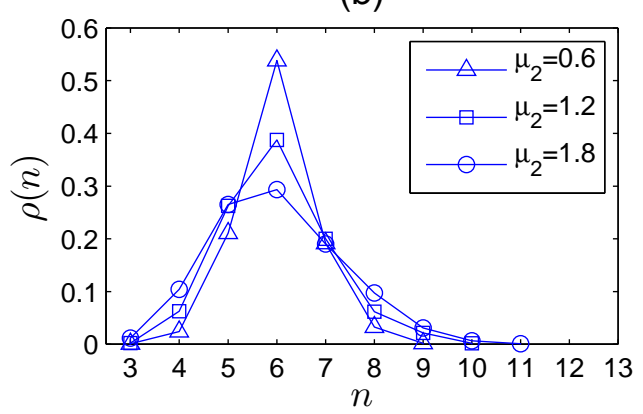

(c)

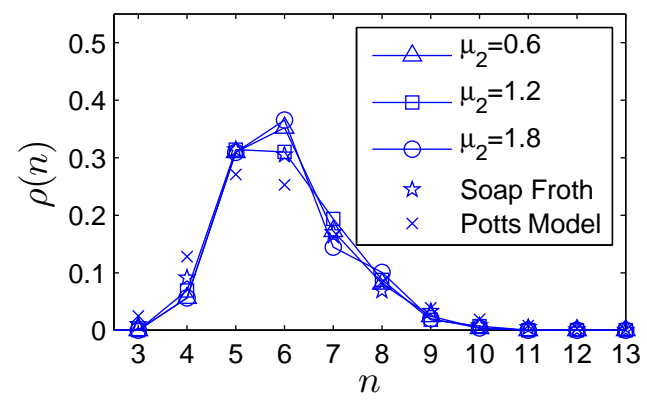

Figure 5: The upper panel shows the second moment $\mu_{2}$ as functions of time for the three different initial second moments: $\mu_{2}=0.6$ (line with $\triangle$ ), 1.2 (line with $\square$ ), and 1.8 (line with $\bigcirc$ ). The asymptotic scaling state is reached when the second moment $\mu_{2}$ stays in some fixed range, $1.3 \pm 0.3$. The lower panels depict the side distribution $\rho(n)$ at $t=0$ (b) and $t=520 \mathrm{~s}(\mathrm{c})$ for the three different cases. Even though the side distributions $\rho(n)$ are initially different (b), they become very similar at later times (c) and comparable to those obtained in soap froth experiments $(\tau)$ and in the Potts model simulations $(x)$.

\section{2 von Neumann, Lewis, and Aboav-Weaire laws}

The von Neumann law (1.1) expresses that the rate of change of the area of a given cell is linear in time. We verified in [21] that the area change of each cell follows the von Neumann law as long as the cell does not experience any topological change. However, when topological changes occur in a cell, the number of edges of that cell changes and so does the variable $n$ in the von Neumann law. Thus, in order to verify the von Neumann law for foam dynamics involving topological changes, it is reasonable to investigate the change of the average area of cells with the same number of edges [27,34].

Fig. 6(a) plots the rates of change of the average area $d\left\langle A_{n}\right\rangle / d t$ of cells with $n$ edges in terms of the number $n$ of cell sides for the three different initial second moments: $\mu_{2}=0.6$ $(\triangle), 1.2(\square)$, and $1.8(\bigcirc)$. Here, we select one particular time $t=520 \mathrm{~s}$ in the scaling state to determine $d\left\langle A_{n}\right\rangle / d t$. However, when we choose a data set at different instants, we obtain almost the same graph as the one shown in Fig. 6(a). We can observe that the rates of change of the average area $d\left\langle A_{n}\right\rangle / d t$ follow well the von Neumann law (1.1) represented by the solid linear line with the diffusion constant $k=\frac{\pi}{3} \gamma M \approx 7.54 \times 10^{-6} \mathrm{~cm}^{2} / \mathrm{s}$ used in our 

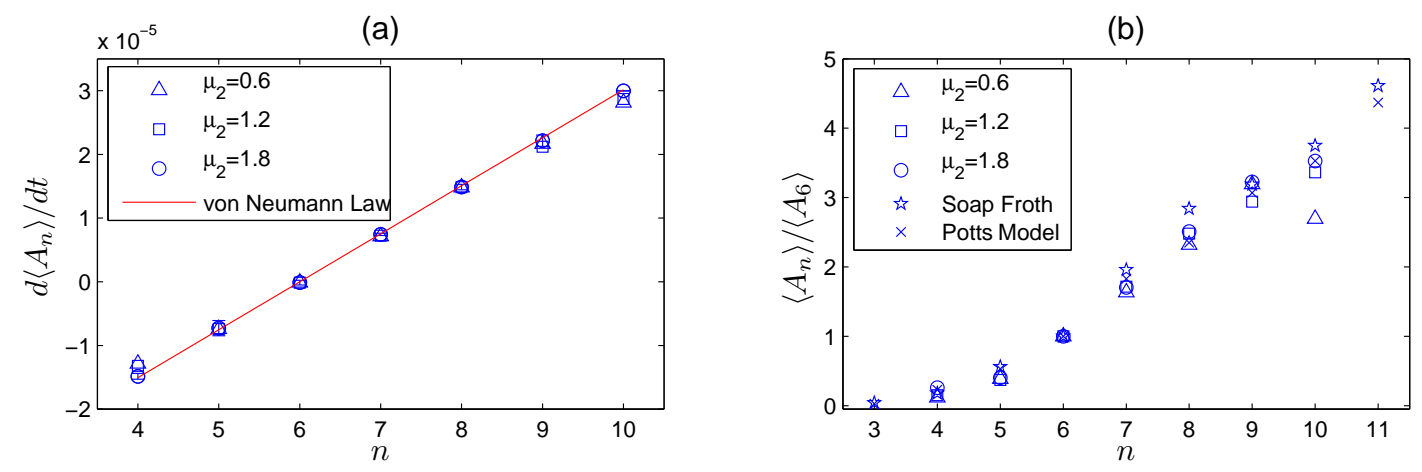

Figure 6: The left panel (a) shows the rates of change of the average area $d\left\langle A_{n}\right\rangle / d t$ of the cells with $n$ sides in terms of the side number $n$ for three different initial second moments: $\mu_{2}=0.6(\triangle), 1.2(\square)$, and $1.8(O)$. The results follow well the von Neumann law (1.1) with the constant $k=\frac{\pi}{3} \gamma M \approx 7.54 \times 10^{-6} \mathrm{~cm}^{2} / \mathrm{s}$ (solid line). Error bars represent the standard deviations of the rate of area change of $n$-sided cells for $\mu_{2}=1.8$. The right panel (b) shows the normalized average area $\left\langle A_{n}\right\rangle /\left\langle A_{6}\right\rangle$ of $n$-sided cells in terms of the side number $n$. Our numerical results are comparable to the data of soap froth experiments $(\xi)$ and Potts model simulations $(x)$, both in [15].

simulations. Error bars representing the standard deviations, which are shown only in the case of $\mu_{2}=1.8$ for clarity since they are similar for the other two cases, are very small.

Lewis $[35,36]$ empirically investigated the average area of $n$-sided cells in twodimensional cellular patterns and found that the average area of $n$-sided cells, $\left\langle A_{n}\right\rangle$, is a linear function of side number $n$ at any fixed time, that is, $\left\langle A_{n}\right\rangle=c_{1}+c_{2} n$, where $c_{1}$ and $c_{2}$ are constants. Fig. $6(\mathrm{~b})$ shows the normalized average area $\left\langle A_{n}\right\rangle /\left\langle A_{6}\right\rangle$ of $n$-sided cells in terms of the side number $n$ for three different initial second moments: $\mu_{2}=0.6(\triangle), 1.2$ $(\square)$, and $1.8(\bigcirc)$. In the scaling state, the normalized average area $\left\langle A_{n}\right\rangle /\left\langle A_{6}\right\rangle$ appears to be independent of the selected time; thus, we choose one particular time $t=520 \mathrm{~s}$ in Fig. $6(\mathrm{~b})$. We can see that the normalized average area $\left\langle A_{n}\right\rangle /\left\langle A_{6}\right\rangle$ is almost linear in the side number $n$ as predicted by Lewis. Moreover, our simulation results agree well with those of soap froth experiments ( $\approx)$ and Potts model simulations $(\times)$ in [15].

In order to understand the distribution of grains in a polycrystal, Aboav empirically investigated the average number of sides of the neighbors, $m(n)$, of $n$-sided cells and suggested the formula $m(n)=5+c / n$, where $c$ is a constant $[37,38]$. Many researchers have further investigated and modified Aboav's formula to introduce the so-called 'AboavWeaire law' $[5,39,40]$ :

$$
m(n)=6-a+\frac{6 a+\mu_{2}}{n}
$$

where $a$ is a constant close to unity, and $\mu_{2}$ is the second moment. This law implies that a cell with a large number of sides has a tendency to be surrounded by cells with a small number of sides, and vice versa.

In Fig. 7, we plot $n \times m(n)$ as functions of $n$ at a chosen time $(t=520 \mathrm{~s})$ in the scaling state for the three different initial second moments: $\mu_{2}=0.6(\triangle), 1.2(\square)$, and $1.8(\bigcirc)$. Our 


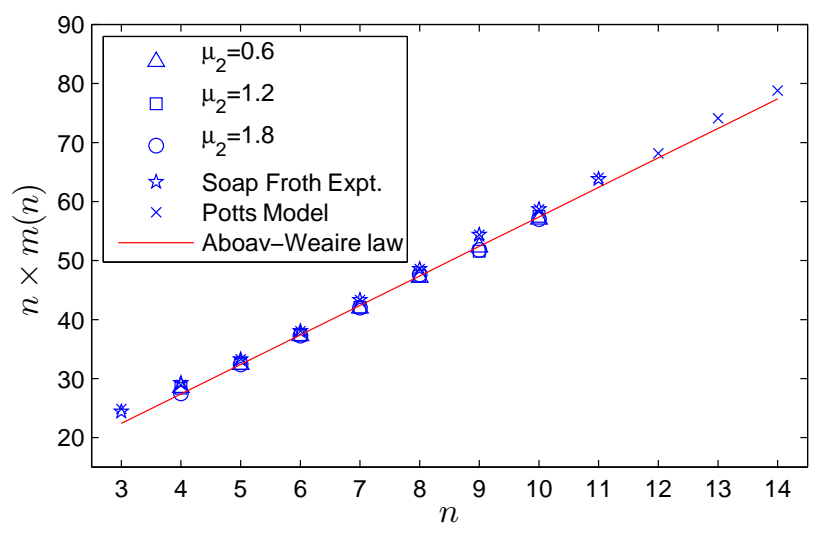

Figure 7: The product of the average number of sides of the neighbors of $n$-sided cells, $m(n)$, and the number $n$ of cell sides, i.e., $n \times m(n)$, in terms of $n\left(\triangle\right.$ for $\mu_{2}=0.6$, $\square$ for $\mu_{2}=1.2$, and $\circ$ for $\left.\mu_{2}=1.8\right)$. Our simulation results are in good agreement with those of soap froth experiments $(\xi)$ and Potts model simulations $(\times)$. The solid line represents the Aboav-Weaire law (3.4) with $a=1.0$ and $\mu_{2}=1.4$.

simulation results are quite comparable to those of soap froth experiments (i) in $[15,30]$, Potts model simulations $(x)$ in [15], and Aboav-Weaire law (3.4) with $a=1.0$ and $\mu_{2}=1.4$ (solid line) in [30]. Note that no cells with three sides or with more than 10 sides are observed in our simulations, as in other studies $[29,41]$.

\subsection{Statistics of foams in dynamic flow}

We here investigate the statistical behaviors of foams under dynamic shear flow which is generated by forcing a rigid ceiling of the region filled with a foam network to slide left and right. Several experimental and numerical studies have found discrepancies in the statistics of foams in dynamic flow and in steady flow [42-44]. These studies were performed with a setting of very slow flow so that the system is assumed to be quasi-static. Since our numerical model uses the full Navier-Stokes equations, the fluid dynamics of a foam is not necessarily restricted to the slow coarsening regime that was considered in the previous subsections.

The rigid ceiling is near the top of the computational domain, $y_{c}=0.9 \mathrm{~cm}$, and moves in an oscillatory manner according to $\mathbf{X}_{w}(s, t)=\left(s+A \sin (2 \pi \omega t), y_{c}\right)$ for $0 \leq s \leq 1.0 \mathrm{~cm}$, where $t$ is the time. The amplitude and the frequency are fixed at $A=2.5 \mathrm{~cm}$ and $\omega=0.004 \mathrm{~Hz}$, respectively. Fig. 8 shows the motion of the foams with the initial second moment $\mu_{2}=1.2$ at some selected times: $t=0,280 \mathrm{~s}, 360 \mathrm{~s}, 440 \mathrm{~s}, 520 \mathrm{~s}$, and $600 \mathrm{~s}$. We also depict the velocity fields for the fluid which indicate that the flow depends on the oscillatory motion of the rigid ceiling as well as the surface tension of the internal foam boundaries. One can observe that the foam goes through both diffusive coarsening and oscillatory shearing motion.

Fig. 9 shows the numbers of T1 processes (see Fig. 1) occurring in each of the time intervals of $12.5 \mathrm{~s}$ for the foams with the initial second moments $\mu_{2}=0.6$ (left) and 1.8 
(a) $t=0$

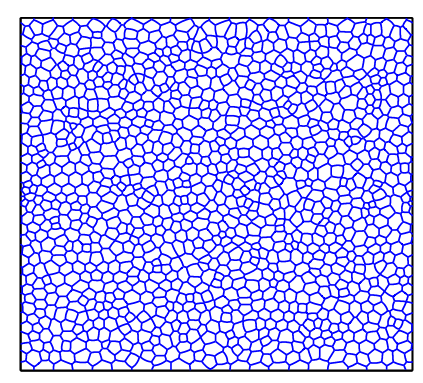

(d) $\mathrm{t}=440 \mathrm{~s}$

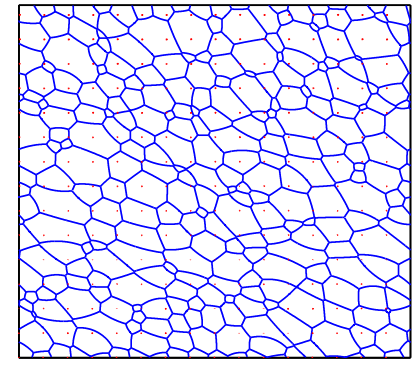

(b) $\mathrm{t}=280 \mathrm{~s}$

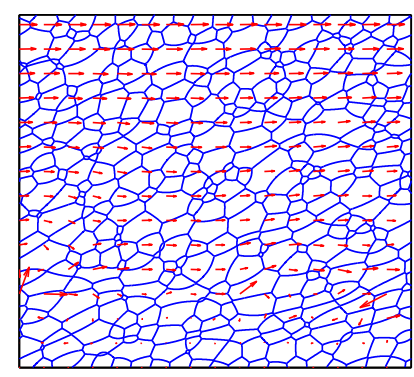

(e) $\mathrm{t}=520 \mathrm{~s}$

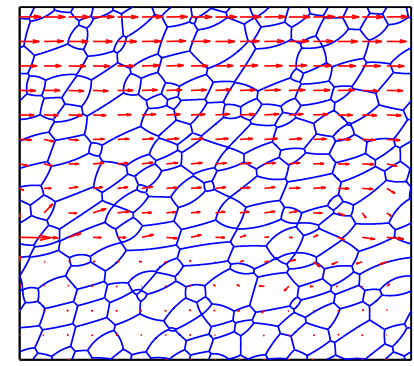

(c) $t=360 \mathrm{~s}$

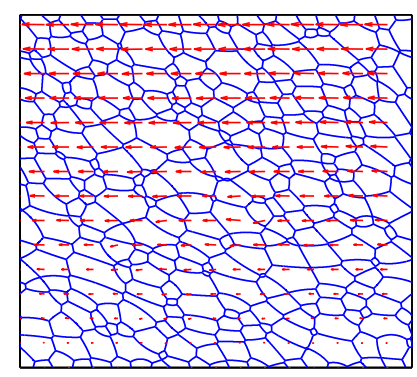

(f) $t=600 \mathrm{~s}$

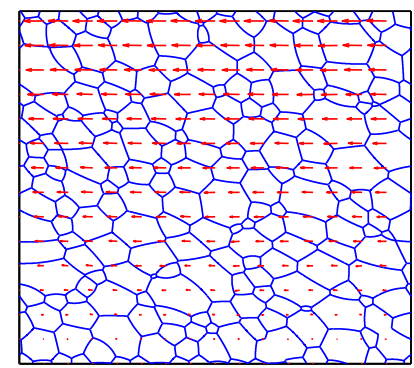

Figure 8: The motion of the foam with the initial second moment $\mu_{2}=1.2$ in an oscillatory shear flow with frequency $\omega=0.004 \mathrm{~Hz}$ and amplitude $A=2.5 \mathrm{~cm}$ at $t=0$ (a), $280 \mathrm{~s}(\mathrm{~b}), 360 \mathrm{~s}(\mathrm{c}), 440 \mathrm{~s}(\mathrm{~d}), 520 \mathrm{~s}(\mathrm{e})$, and $600 \mathrm{~s}(\mathrm{f})$. The arrows represent the velocity fields of the background fluid. The foam experiences both diffusive coarsening and shearing motion due to the oscillatory motion of the rigid ceiling.

(right) in the static (top) and dynamic shear (bottom) flows. We can see that the T1 processes occur more frequently in the dynamic flow than in the static flow so that the total numbers of T1 processes during $800 \mathrm{~s}$ are 1579 in the case (a), 1465 in the case (b), 7342 in the case (c), and 5809 in the case (d). Moreover, in the dynamic flow, the numbers of T1 processes behave in the same oscillatory manner as the shear rate which is proportional to the velocity of the ceiling, $2 \pi \omega A \cos (2 \pi \omega t)$ which is drawn in the solid lines in (c) and (d). This confirms the experimental and numerical observations that, when the shear rate increases, the rate of $\mathrm{T} 1$ processes (rearrangements) increases [45, 46].

It is interesting to note that, even though the rates of $\mathrm{T} 1$ processes are different in the static and dynamic shear flows, the number of $\mathrm{T} 2$ processes is almost the same during the simulation time in both cases, which are approximately 1040. This results in almost identical numbers of cells and identical average cell sizes in static and dynamic shear flows, which might give the evidence that the shear has minimal effect on foam structure and on the coarsening processes $[43,45]$.

Fig. 10 shows some statistical behaviors of the foams with three different initial second moments $\left(\mu_{2}=0.6,1.2\right.$, and 1.8) in the dynamic flow. The total numbers of cells which are shown in (a) decrease in time, indicating the coarsening process of the foams. The decreasing speed of the cell number is proportional to the initial complexity for some 
(a) static with $\mu_{2}=0.6$

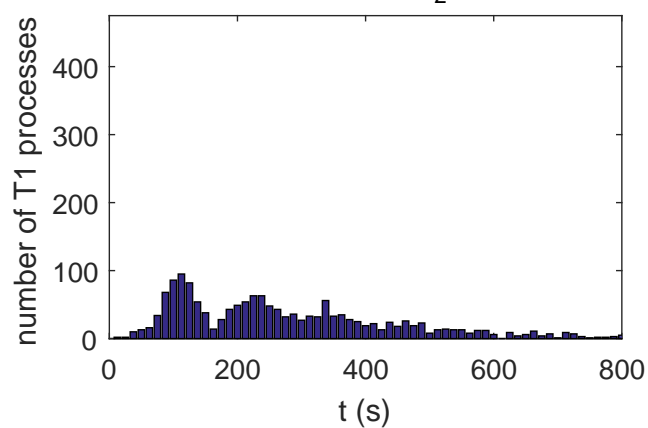

(c) dynamic with $\mu_{2}=0.6$

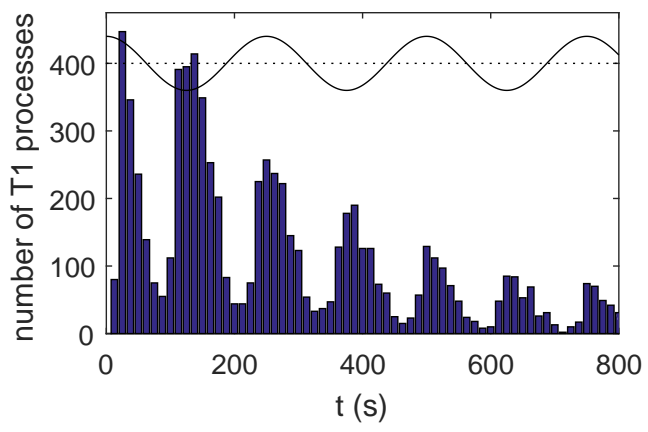

(b) static with $\mu_{2}=1.8$

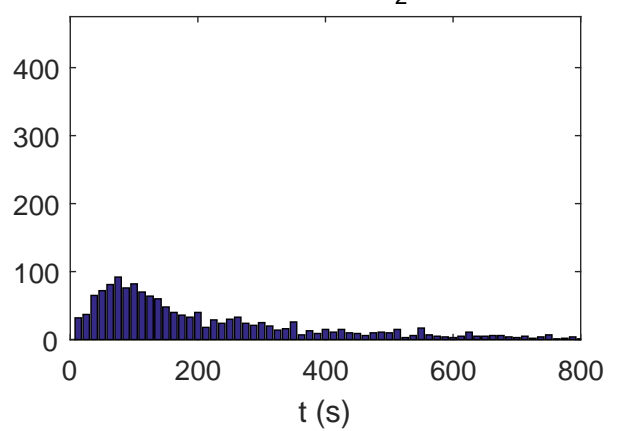

(d) dynamic with $\mu_{2}=1.8$

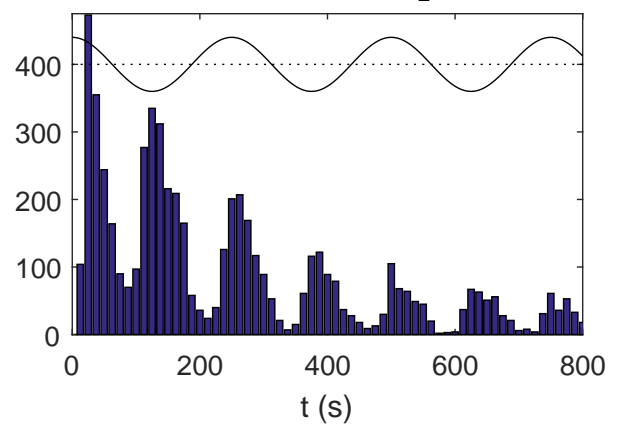

Figure 9: The numbers of T1 processes occurring in each of the time interval of $12.5 \mathrm{~s}$ for the foams with the initial second moments $\mu_{2}=0.6$ (left) and 1.8 (right) in the static (top) and dynamic shear (bottom) flows. The $\mathrm{T} 1$ processes occur more frequently in the dynamic flow than in the static flow, and the number of T1 processes is proportional to the shear strain rate indicated by the solid lines in $(c)$ and $(d)$.

time; however, the total numbers of cells are similar for all three cases after about $t=600 \mathrm{~s}$. These coarsening behaviors are similar to those of the foams in the steady flow, compare with Fig. 3(a).

Fig. 10(b) shows the second moment $\mu_{2}$ as functions of time. The second moment $\mu_{2}$ starts at different values but oscillates around some fixed range $1.8 \pm 0.4$ after some transient time even in the dynamic flow considered here. The range in which the second moment of side distribution stays is larger than those in the case of the steady flow and in literature, compare with Fig. 5(a). Whereas a limiting scaling state exists even in dynamic flow, the foam structures are more complex and changeable due to the background shear flow. We also have observed that the side distribution $\rho(n)$ varies slightly more in time in the dynamic flow than in the steady flow; however, it generally agrees well with those obtained by the soap froth experiments ( $\lesssim$ ) and by the Potts model simulations $(x)$ as shown in Fig. 10(c).

When the foams are in limiting scaling states, Fig. 10 depicts the rate of change of the average area of the $n$-sided cells, $d\left\langle A_{n}\right\rangle / d t(\mathrm{~d})$, the normalized average area of $n$-sided cells, $\left\langle A_{n}\right\rangle /\left\langle A_{6}\right\rangle$ (e), and the product of the average number of sides of the neighbors of $n$-sided cells and the side number $n, n \times m(n)(\mathrm{f})$, in terms of the side number $n$. Even 
(a)

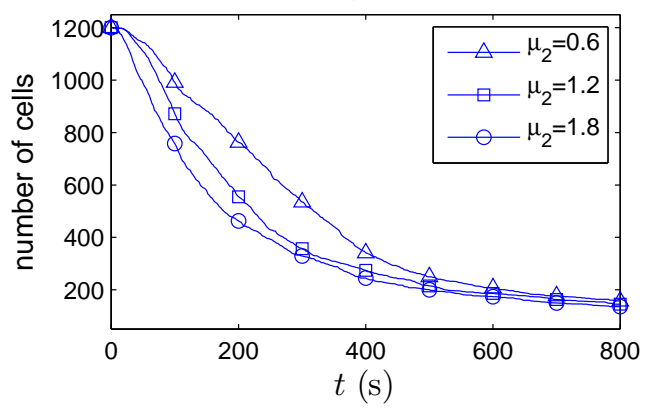

(c)

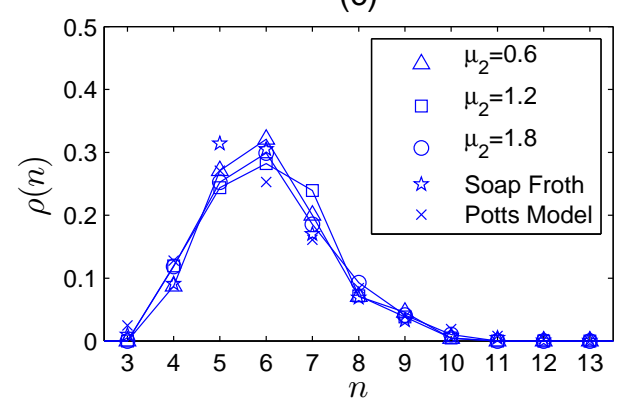

(e)

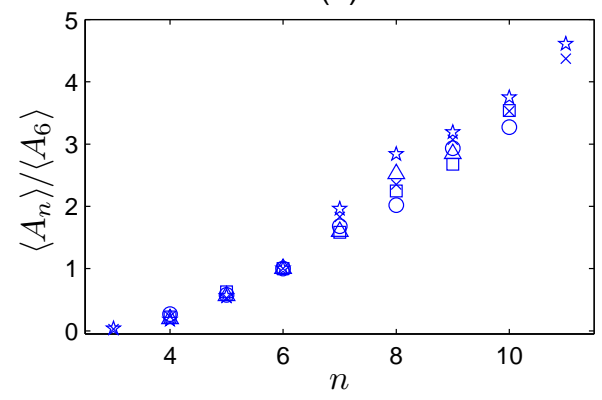

(b)

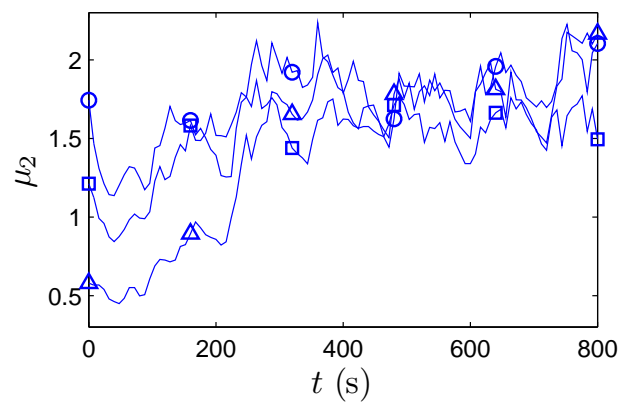

(d)

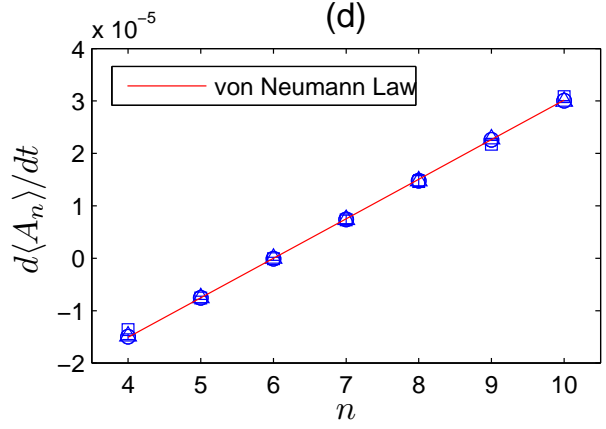

(f)

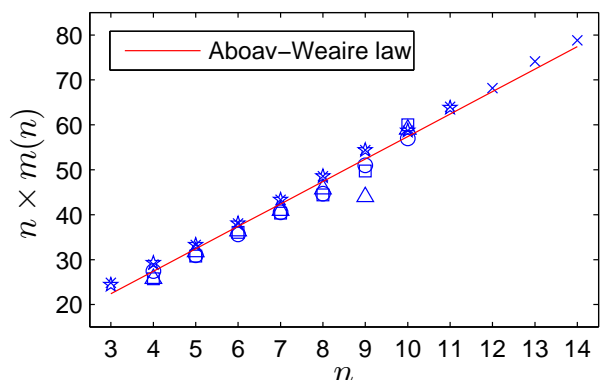

Figure 10: The total number of cells (a) and the second moment $\mu_{2}(\mathrm{~b})$ are drawn as functions of time. The side distribution $\rho(n)(\mathrm{c})$, the rate of change of the average area of the $n$-sided cells, $d\left\langle A_{n}\right\rangle / d t$ (d), the normalized average area of $n$-sided cells, $\left\langle A_{n}\right\rangle /\left\langle A_{6}\right\rangle(\mathrm{e})$, and $n \times m(n)(\mathrm{f})$ where $m(n)$ is the average number of sides of the neighbors of $n$-sided cells are depicted in terms of the side number $n$ at $t=520 \mathrm{~s}$. The foams have three different initial second moments $\left(\mu_{2}=0.6,1,2\right.$, and 1.8$)$ in an oscillatory shear flow. Even in the dynamic shear flow, the statistical behaviors of the foams shown here are qualitatively similar to those in steady flow.

though the foam is now far from equilibrium, these quantities verify well von Neumann relation (1.1) (d), Lewis law (e), and Aboav-Weaire law (3.4) (f).

It is interesting to find the reason why the von Neumann relation holds well even in a dynamic flow which may deform the internal boundaries of a foam in some complicated way, so that the curvature of each edge of the cells vary along the edge. In the derivation of von Neumann relation which is given in a discretized setting in Appendix, it is crucial 
(a)

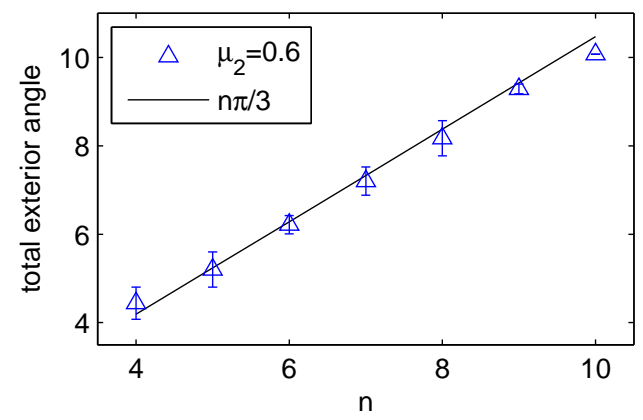

(b)

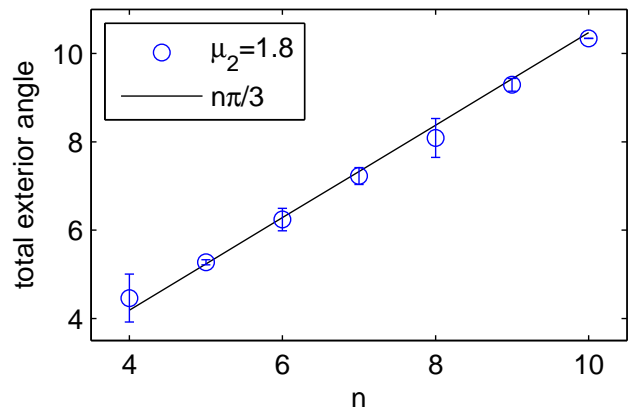

Figure 11: The average value of total exterior angles with the standard deviations as functions of the number $n$ of edges at $t=520 \mathrm{~s}$ for the two cases with $\mu_{2}=0.6$ (a) and 1.8 (b). The slope of the total exterior angle in the dynamic flow is linear with the slope $\pi / 3$ which is assumed in theory (solid line).

that the following two conditions satisfy: (1) the background fluid is incompressible, i.e., $\nabla \cdot \mathbf{u}=0$, which implies that the fluid (steady or dynamic) makes no contribution to the area change of a region enclosed by edges of a given cell, and (2) three edges meet at junction points with the equal angle $(2 \pi / 3)$, which makes the total exterior angle to be $n \pi / 3$ for $n$-edged cell. See Appendix for the detailed derivation of von Neumann relation in a $2 \mathrm{D}$ discretized setting.

Fig. 11 shows the average value of total exterior angles with the standard deviations as functions of the number of edges $n$ at $t=520 \mathrm{~s}$ for the two cases with initial $\mu_{2}=0.6$ (a) and 1.8 (b). We can see that the total exterior angles agree well with $n \pi / 3$ (solid line) assumed in theory. Thus, together with the incompressibility of the fluid, von Neumann relation is well satisfied even in the dynamical flow.

\section{Summary and conclusions}

In this paper, we have investigated the statistical behaviors of two-dimensional dry foam using numerical simulations based on the immersed boundary method. We modeled the liquid phase of a foam as a thin elastic boundary with surface tension and the gas phase as a viscous incompressible fluid which can go through the liquid boundary. We have also described an algorithm for topological change of a foam network using the combination of T1 and T2 processes and computed statistical data in the coarsening process of the foam.

We have shown some statistical data of foams such as the total number of cells, average area, side distribution, and second moment of the distribution of the numbers of cell sides. These statistical data give evidence of the existence of a limiting scaling state for the dry foam dynamics in which the asymptotic value of the second moment $\mu_{2}$ lies in the range of $1.3 \pm 0.3$. We have also numerically verified some well-known formulas in two-dimensional dry foam such as von Neumann relation, Lewis law, and Aboav-Weaire 
law, together with the comparison of our simulation results with those of experiments and other simulations.

In addition, we have investigated the statistical behaviors of two-dimensional dry foam in an oscillatory shear flow, and found that the statistical behaviors of foam coarsening in a dynamic flow are similar to those in the steady flow. Especially, our simulation results show that there exists a limiting scaling state of the dry foam in a dynamic flow in which the asymptotic value of $\mu_{2}$ lies in the range $1.8 \pm 0.4$. Even in this dynamic flow, the von Neumann, Lewis, and Aboav-Weaire laws are well satisfied.

\section{Acknowledgments}

Y. Kim was supported by a National Research Foundation of Korea grant funded by the Korean government (Grant No. 2017R1E1A1A03070636).

\section{Appendix: von Neumann relation in a 2D discretized setting}

In this appendix, we derive a discretized version of von Neumann relation [2] for the rate of change of area of a cell within a dry foam. We give the details to point out the relationship to our scheme, and to highlight the various approximations that are made along the way. The errors in these approximations should approach zero as the resolution of the computation is refined.

For simplicity, we begin by considering an isolated cell, i.e., a soap film in the form of a closed curve that partitions $\mathbb{R}^{2}$ into an interior region and an exterior region, see the left panel of Fig. 12. In a discretized setting, the isolated cell denoted as $C(t)$ is expressed as a polygon with its vertices being marker points, see the right panel of Fig. 12. Let $\mathbf{X}_{k}$, $k=0, \cdots, N_{b}$, represent the vertices of the polygonal cell and $L_{k}$ represent the line segment connecting $\mathbf{X}_{k}$ and $\mathbf{X}_{k+1}$. Since the cell is a closed curve, $\mathbf{X}_{0}=\mathbf{X}_{N_{b}}$. Then it is straightforward to show that

$$
\frac{d A}{d t}=\sum_{k=0}^{N_{b}-1} \frac{1}{2}\left(\left|L_{k}\right| \mathbf{n}_{k}+\left|L_{k-1}\right| \mathbf{n}_{k-1}\right) \cdot \frac{d \mathbf{X}_{k}}{d t},
$$

where $A(t)$ is the area of the polygonal cell, $\left|L_{k}\right|$ is the length of the line segment $L_{k}$, and $\mathbf{n}_{k}$ is the unit normal to the line segment $L_{k}$ pointing toward the outside of the polygon.

Our formula for $d \mathbf{X}_{k} / d t$ can be given by approximating Eq. (3.3) as

$$
\frac{d \mathbf{X}_{k}}{d t}=\mathbf{U}_{k}+M \frac{\mathbf{F}_{k} \Delta s}{\frac{1}{2}\left(\left|L_{k}\right|+\left|L_{k-1}\right|\right)}
$$

where $\mathbf{U}_{k}$ is the interpolated fluid velocity evaluated at $\mathbf{X}_{k}$. Here, the force density is derived from the relation $\mathbf{F}_{k} \Delta s=-\partial E / \partial \mathbf{X}_{k}$, where $E[\mathbf{X}]$ is the discretized energy functional 

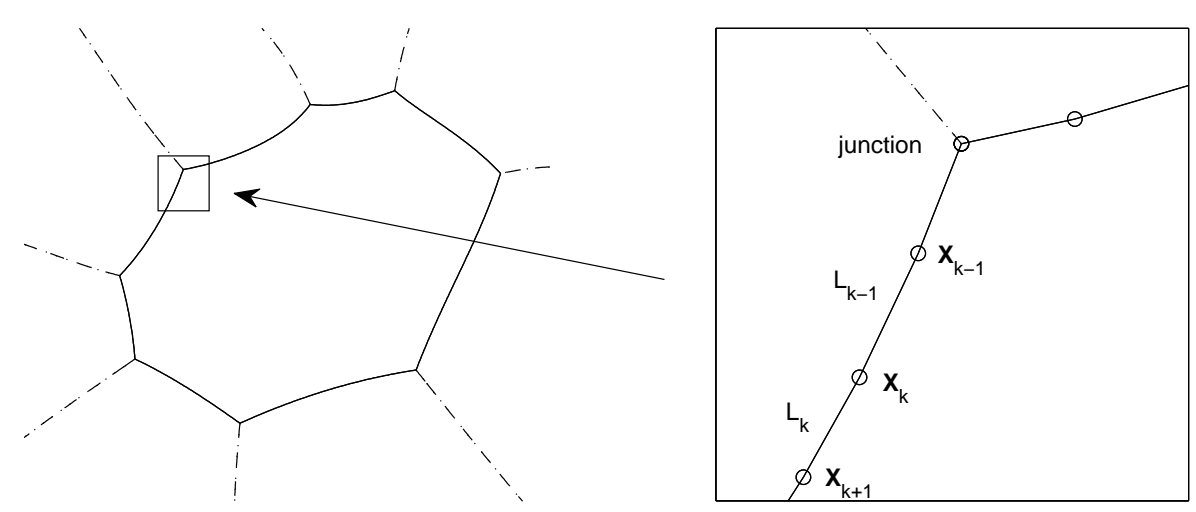

Figure 12: An isolated cell with 8 edges in a 2D discretized setting is expressed as a polygon with its vertices being marker points $\mathbf{X}_{k}, k=0, \cdots, N_{b}$ and with the edges being line segments $L_{k}$ connecting $\mathbf{X}_{k}$ and $\mathbf{X}_{k+1}$. There are special vertices called junction points, at which three edges meet and there is no permeability.

of the form $E[\mathbf{X}]=\gamma \sum_{i=1}^{N_{b}-1}\left|L_{i}\right|$ and has the following expression:

$$
\begin{aligned}
\mathbf{F}_{k} \Delta s & =\gamma\left(\frac{\mathbf{X}_{k+1}-\mathbf{X}_{k}}{\left|L_{k}\right|}-\frac{\mathbf{X}_{k}-\mathbf{X}_{k-1}}{\left|L_{k-1}\right|}\right) \\
& =\gamma\left(\boldsymbol{\tau}_{k}-\boldsymbol{\tau}_{k-1}\right)
\end{aligned}
$$

where $\boldsymbol{\tau}_{k}=\left(\mathbf{X}_{k+1}-\mathbf{X}_{k}\right) /\left|L_{k}\right|$ is the unit tangent in the direction from $\mathbf{X}_{k}$ to $\mathbf{X}_{k+1}$.

We now substitute (A.2) and (A.3) into (A.1), but we make the following approximation

$$
\sum_{k=1}^{N_{b}-1} \frac{1}{2}\left(\left|L_{k}\right| \mathbf{n}_{k}+\left|L_{k-1}\right| \mathbf{n}_{k-1}\right) \cdot \mathbf{U}_{k}=0 .
$$

This is a discretization of $\int_{C(t)} \mathbf{u} \cdot \mathbf{n} d l$, which is 0 since $\nabla \cdot \mathbf{u}=0$. With this approximation, Eq. (A.1) becomes

$$
\frac{d A}{d t}=M \gamma \sum_{k=1}^{N_{b}-1} \frac{1}{2}\left(\left|L_{k}\right| \mathbf{n}_{k}+\left|L_{k-1}\right| \mathbf{n}_{k-1}\right) \cdot \frac{\boldsymbol{\tau}_{k}-\boldsymbol{\tau}_{k-1}}{\frac{1}{2}\left(\left|L_{k}\right|+\left|L_{k-1}\right|\right)} .
$$

Let us define $\theta_{k}$ to be the angle of the vector $\tau_{k}$ from the positive $x$-axis, then $\tau_{k}=$ $\left(\cos \left(\theta_{k}\right), \sin \left(\theta_{k}\right)\right)$ and $\mathbf{n}_{k}=\left(\sin \left(\theta_{k}\right),-\cos \left(\theta_{k}\right)\right)$. Using the relations $\mathbf{n}_{k-1} \cdot \boldsymbol{\tau}_{k}=-\sin \left(\theta_{k}-\theta_{k-1}\right)$ and $\mathbf{n}_{k} \cdot \boldsymbol{\tau}_{k-1}=\sin \left(\theta_{k}-\theta_{k-1}\right)$, we can simplify Eq. (A.5) to obtain

$$
\frac{d A}{d t}=-M \gamma \sum_{k=1}^{N_{b}-1} \sin \left(\theta_{k}-\theta_{k-1}\right)
$$

In this derivation, we have considered an isolated cell. For a cell in a foam, there are special vertices called junction points, at which three (or possibly more) edges meet (see 
Fig. 12). In our scheme, there is no permeability at junction points, and thus it requires to remove the contribution at the junction points to the summation in Eq. (A.6). Let $J$ be the set of junction points and $E$ be the set of the other vertices, then Eq. (A.6) should be modified to

$$
\frac{d A}{d t}=-M \gamma \sum_{k \in E} \sin \left(\theta_{k}-\theta_{k-1}\right) .
$$

At the vertices in $E$, we can make the approximation that $\sin \left(\theta_{k}-\theta_{k-1}\right) \approx\left(\theta_{k}-\theta_{k-1}\right)$. This approximation improves along with the resolution of the computation, provided that the polygonal curve approaches a smooth curve as the discretization is refined. The von Neumann law is derived using the assumption that three edges of a foam meet at a junction point with the equal angles, which are $2 \pi / 3$. Thus, at the vertex $\mathbf{X}_{k}$ in $J$, the exterior angle $\theta_{k}-\theta_{k-1}$ is $\pi / 3$. With these assumption and approximation, we can derive the $2 \mathrm{D}$ von Neumann relation in the discretized setting:

$$
\begin{aligned}
\frac{d A}{d t} & =-M \gamma \sum_{k \in E}\left(\theta_{k}-\theta_{k-1}\right) \\
& =-M \gamma \sum_{k \in J \cup E}\left(\theta_{k}-\theta_{k-1}\right)+M \gamma \sum_{k \in J} \frac{\pi}{3} \\
& =-2 \pi M \gamma+M \gamma \frac{n \pi}{3},
\end{aligned}
$$

where $n$ is the number of junction points of the cell. In the third equality, we use the fact that a polygon has the total exterior angle $2 \pi$.

\section{References}

[1] D. Weaire and S. Hutzler, The Physics of Foams, Oxford University Press, 1999.

[2] J. von Neumann, Grain shapes and other metallurgical applications of topology. J. in Metal Interfaces (ed. C.Herring), 51:108-110, 1951.

[3] W.W. Mullins, Two-dimensional motion of idealized grain boundaries. J. Appl. Phys. 27:900904, 1956.

[4] C.S. Smith, Grain shapes and other metallurgical applications of topology. J. in Metal Interfaces (ed. C.Herring), 51:65-108, 1952.

[5] J.A. Glazier, The Dynamics of Two Dimensional Cellular Patterns. Ph.D. thesis, University of Chicago, 1989.

[6] J.P. Kermode and D. Weaire, 2D-Froth: a program for the investigation of 2-dimensional froths. Computer Physics Communications 60, 75-109, 1990.

[7] J. Duplat, B. Bossa, and E. Villermaux, On two-dimensional foam ageing. J. Fluid Mech. 673:147-179, 2011.

[8] K.Y. Szeto and W.Y. Tam, Universal topological properties of shells in soap froth. Phy. Rev. E, 53:4213-4216, 1996.

[9] C. Quilliet, S. A. Talebi, D. Rabaud, J. Käfer, S.J. Cox, and F. Graner, Topological and geometrical disorders correlate robustly in two-dimensional foams. Phil. Mag. Lett. 88:651-660, 2008. 
[10] H.J. Ruskin and Y. Feng, Scaling properties for ordered/disordered 2-D dry froths. Physica A, 230:455-466, 1996.

[11] J.J. Chae and M. Tabor, Dynamics of foams with and without wall rupture. Phy. Rev. E, 55:598-610, 1997.

[12] M. Durand, A.M. Kraynik, F. van Swol, J. Käfer, C. Quilliet, S. Cox, S.A. Talebi, and F. Graner, Statistical mechanics of two-dimensional shuffled foams: Geometry-topology correlation in small or large disorder limits. Phy. Rev. E, 89, 2014.

[13] D. Weaire and J.P. Kermode, Computer simulation of a two-dimensional soap froth II. Analysis of results. Phil. Mag. B. 50:379-395, 1984.

[14] T. Herdtle and H. Aref, Numerical experiments on two-dimensional foam. J. Fluid Mech. 241:233-260, 1992.

[15] J.A. Glazier, M.P Anderson, and G.S. Grest, Coarsening in the two-dimensional soap froth and the large-Q Potts model: a detailed comparison. Phil. Mag. B. 62:615-645, 1990,

[16] I. Fortuna, G.L. Thomas, and R.M.C. Almeida, Growth laws and self-similar growth regimes of coarsening two-dimensional foams: transition from dry to wet limits. Phys. Rev. Lett. 108, 2012.

[17] X. Li, H. Zhou, and C. Pozrikidis, A numerical study of the shearing motion of emulsions and foams. J. Fluid Mech. 286:379-404, 1995.

[18] I.B. Bazhlekov, P.D. Anderson, and H.E.H. Meijer, Nonsingular boundary integral method for deformable drops in viscous flows. Phys. Fluids, 16(4), 2004.

[19] H.J. Frost and C.V. Thompson, Computer simulation of grain growth. Current Opinion in Solid State and Materials Science, 1:361-368, 1996.

[20] A.M. Kraynik and D.A. Reinelt, Foam microrheology: from honeycombs to random foams. Proceedings of the PPS-15, 1999.

[21] Y. Kim, M.-C. Lai, and C.S. Peskin, Numerical simulations of two-dimensional foam by the immersed boundary method. J. Comput. Phys. 229:5194-5207, 2010.

[22] C.S.Peskin, The immersed boundary method. Acta Numerica, 11:479-517, 2002.

[23] Y. Kim and C.S. Peskin, 2-D parachute simulation by the Immersed Boundary Method. SIAM J.Sci.Comput. 28(6), 2006.

[24] Y. Kim, Y. Seol, M.-C. Lai, and C.S. Peskin, The immersed boundary method for twodimensional foam with topological changes. Commun. Comput. Phys. 12:479-493, 2012.

[25] J.A. Glazier and D. Weaire, The kinetics of cellular patterns. J. Phys.: Condens. Matter, 4:1867-1894, 1992.

[26] J.A. Glazier, S.P. Gross, and J. Stavans, Dynamics of two-dimensional soap froths. Phys. Rev. A, 36:306-312, 1987.

[27] M. Marder, Soap-bubble growth. Phys. Rev. A, 438(36), 1987.

[28] W.Y. Tam and K.Y. Szeto, Evolution of soap froth under temperature effects. Phys. Rev. E. 53:877-880, 1996.

[29] A.E. Roth, C.D. Jones, and D.J. Durian, Bubble statistics and coarsening dynamics for quasitwo-dimensional foams with increasing liquid content. Phy. Rev. E, 87, 2013.

[30] J. Stavans and J.A. Glazier, Soap Froth Revisited: Dynamic Scaling in the Two-Dimensional Froth. Phys. Rev. Lett, 62:1318-1321, 1989.

[31] T. Trittel, T. John, and R. Stannarius, Smectic Foams. Langmuir, 26:7899-7904, 2010.

[32] D. Weaire and H. Lei, A Note on the Statistics of the Mature 2-Dimensional Soap Froth. Phil. Mag. Lett. 62: 427-430, 1990.

[33] T. Herdtle and H. Aref, Numerical experiments on two-dimensional foam. J. Fluid Mech. 241:233-260, 1992. 
[34] D. Fan, C. Geng, and L.-Q. Chen, Computer simulation of topological evolution in 2-D grain growth using a continuum diffuse-interface field model. Acta Mater. 45:1115-1126, 1997.

[35] F.T. Lewis, The correlation between cell division and the shapes and sizes of prismatic cells in the epidermis of Cucumis. Anat. Rec. 38: 341-376, 1928.

[36] F.T. Lewis, The correlation between cell division and the shapes and sizes of prismatic cells in the epidermis of Cucumis. Am. J. Bot. 31:619, 1944.

[37] D.A. Aboav, The arrangement of grains in a polycrystal. Metallography. 3:383-390, 1970.

[38] D.A. Aboav, The arrangement of cells in a net. II. Metallography, 16:265-273, 1983.

[39] C.J. Lambert and D. Weaire, Some remarks on the arrangement of grains in a polycrystal. Metallography. 14: 307-318, 1981.

[40] N. Rivier, Statistical crystallography: structure of random cellular networks. Philos. Mag. B. 52:795-819, 1985.

[41] D. Weaire and J.P. Kermode, Computer simulation of a two-dimensional soap froth I. Method and motivation. Phil. Mag. B. 48:245-259, 1983.

[42] T. Herdtle, Numerical Studies of Foam Dynamics. Ph.D. thesis, University of California, San Diego, 1991.

[43] D. Weaire, F. Bolton, T. Herdtle, and H. Aref, The effect of strain upon the topology of a soap froth. Phil. Mag. Lett. 66:293-299, 1992.

[44] A. Adb el Kader and J.C. Earnshaw, Shear-induced chagnes in two-dimensional foam. Phy. Rev. Lett. 82:2610-2613, 1999.

[45] A.D. Gopal and D.J. Durian, Nonlinear Bubble Dynamics in a Slowly Driven Foam. Phys. Rev. Lett. 75:2610-2613, 1995.

[46] A.D. Gopal and D.J. Durian, Relaxing in Foam. Phys. Rev. Lett. 91(18), 2003. 